\title{
Research Note \\ On the broadening of emission lines in active galactic nuclei
}

\author{
L. Foschini ${ }^{\star}$ \\ Istituto di Astrofisica Spaziale e Fisica Cosmica (IASF-CNR), Sezione di Bologna ${ }^{\star \star, ~ V i a ~ G o b e t t i ~ 101, ~}$ \\ 40129 Bologna, Italy \\ Received 4 July 2001 / Accepted 4 February 2002

\begin{abstract}
Recent works, both theoretical and observational, have suggested that turbulence could play a nonnegligible role in the broadening of emission lines in active galactic nuclei. The purpose of this note is to show
\end{abstract} \\ how shock wave-turbulence interaction, under unsteady regimes, can affect the broadening of emission lines.
}

Key words. galaxies: active - shock waves - turbulence - line: formation - line: profiles

\section{Introduction}

The Seyfert galaxies are a class of active galactic nuclei (AGN) characterized by nuclear spectra with strong emission lines generated by highly ionized atoms. These lines are of two types: narrow, corresponding to low density plasma with bulk velocities of the order of $100 \mathrm{~km} \mathrm{~s}^{-1}$ and broad, corresponding to high density plasma and bulk velocities of the order of $10^{4} \mathrm{~km} \mathrm{~s}^{-1}$. The main physical mechanism responsible for the ionization is the non-stellar photon flux coming from the AGN itself (for reviews, see Davidson \& Netzer 1979; Krolik 1999; Fabian et al. 2000; Sulentic et al. 2000). However, also shocks can provide the physical conditions necessary to the ionization of the atoms. This occurs, for example, when the postshock temperature reaches values higher than $1 \mathrm{eV}$, and therefore the shocked gas emits a continuum in the EUV-soft $\mathrm{X}_{-}$ ray band, which is quickly absorbed by nearby gas and its energy converted into emission lines. See also, however, the work of Perry \& Dyson (1985), Collin-Souffrin et al. (1988), the former recently updated and enlarged by Fromerth \& Melia (2001).

The main objection to shock formation of line emission is that the wide range of observed ionization stages is not fully compatible with a thermal emission from post-shock regions. The temperature required to produce the highest stages are far from those obtained in shocks. In addition, according to Krolik (1999), we have no direct evidence of the existence of shocks, but other authors suggested that shocks can be important in the morphology, kinematics, and excitation of emission lines in Seyfert galaxies (Colbert et al. 1998; Allen et al. 1999). Indeed, the emis-

* e-mail: foschini@tesre.bo.cnr.it

$\star \star$ Formerly Institute TeSRE - CNR. sion line ratios do not demonstrate the excitation mechanism (shocks or otherwise). Moreover, the velocity field and line width are consistent with shocks and with energetics determined from radio and X-ray observations (Allen et al. 1999).

Turbulence has been invoked to explain the line broadening (Horne 1995; Pariev \& Bromley 1998), but some authors object that the turbulent velocity field in an AGN disk is not so strong to affect the line profile (Fabian et al. 2000). Bottorff et al. (2000) have found that extra thermal, non-dissipative microturbulence somewhere between 0 and $10^{3} \mathrm{~km} \mathrm{~s}^{-1}$ is consistent with observations of a small sample of quasars with high signal to noise spectra (Baldwin et al. 1996). In addition, Bottorff \& Ferland (2002) find that $\approx 200 \mathrm{~km} \mathrm{~s}^{-1}$ microturbulence with dissipation is able to explain typical emission line ratios found in quasars and other AGN. Observations poorly constrain the range of turbulence in AGN, but even the possibility of its presence has important ramifications in the analysis of AGN emission lines. It is therefore useful to investigate mechanisms that generate and sustain (possibly high levels of) turbulence.

The purpose of this note is to discuss how the interplay between shock waves and turbulence can enhance the turbulent velocity field. These physical issues have been already applied to the line broadening in pulsating stars (Gillet et al. 1998) and we try to apply these concepts to active galactic nuclei.

\section{Fully developed turbulence}

We note here some concepts of fully developed turbulence. We refer to Landau \& Lifshitz (1987). Turbulent flow is characterized by random variations in space and time 
in the velocity field and develops at very high Reynolds number:

$R e=\frac{l V \rho}{\mu}$

where $l$ is the characteristic dimension of the flow, $V$ is the velocity, $\rho$ the mass density, and $\mu$ the viscosity, which is equal to about $10^{-17} T^{5 / 2} \mathrm{~g} \cdot \mathrm{cm}^{-1} \mathrm{~s}^{-1}$ for a fully ionized unmagnetized hydrogen plasma (see Choudhuri 1998). For a typical electron temperature of $10^{4} \mathrm{~K}$, we have $\mu=$ $10^{-7} \mathrm{~g} \cdot \mathrm{cm}^{-1} \mathrm{~s}^{-1}$.

By considering a typical flow speed of $V_{2} \mathrm{~km} \mathrm{~s}^{-1}$, where $V_{2}=V / 100 \mathrm{~km} \mathrm{~s}^{-1}$, and a mean density of $1.67 \times$ $10^{-15} \mathrm{~g} / \mathrm{cm}^{3}$ (i.e. an electron volume density of $10^{9} \mathrm{~cm}^{-3}$ for a fully ionized hydrogen plasma), we obtain from Eq. (1) that $R e \approx 0.167 \cdot l \cdot V_{2}$, where $l$ is in $\mathrm{cm}$. Therefore, when the characteristic dimension of the flow is of some hundreds of metres we have already a turbulent flow (for a speed of the order of $100 \mathrm{~km} \mathrm{~s}^{-1}$ ). On astrophysical scales, we can consider that the characteristic length is of the order of astronomical units (AU), so that the flow is fully turbulent, even though in presence of tiny values of viscosity.

In a turbulent regime, we can divide the velocity $\boldsymbol{V}$ into a mean value component $\overline{\boldsymbol{v}}$, obtained from an average over a long time, plus a fluctuating part $\boldsymbol{u}$ that when averaged is equal to zero:

$\boldsymbol{V}=\overline{\boldsymbol{v}}+\boldsymbol{u}$.

The order of magnitude of the distance over which the speed varies appreciably (i.e., the dimension of the largest eddies) has the same order of the dimension $l$ of the region where there is the flow. Therefore, the fluctuation of the speed is of the same order of the variation of the mean speed over the scale $l$. However, under normal conditions, the speed fluctuations cannot exceed the local sound speed $c_{\mathrm{s}}$ (cf. Balbus \& Hawley 1998). With respect to time, the velocity does not vary appreciably over a timescale smaller than about $l / \bar{v}$.

Since the viscosity decreases as the Reynolds number increases, in larger eddies there is no or negligible viscosity, and hence no energy dissipation. The energy flows from larger to smaller eddies, where it is transformed into heat and dissipated. To maintain a steady state flow, an external source of energy must be provided to supply energy to large eddies.

In the plasma of an accretion disk around a supermassive black hole, in addition to the fluid viscosity, other effects take place, because of the nature of the plasma. In this case, the electromagnetic forces play an important role in transferring energy and momentum. The change in state, particularly during the passage of a shock, derives from the collective interactions between charged particles and electromagnetic fields. The fields can be mainly of two types: constant in time (i.e. produced by space charge separation, currents, or other external sources) or fluctuating in time (i.e. produced by plasma instabilities). The first case is usually referred to as laminar, while the second as turbulent. For more details see, for example, Tidman \& Krall (1971).

\section{Shock waves-turbulence interaction}

Despite its importance in several application of science and engineering, the interaction of shock waves with turbulence is still largely unknown. The first problem is to obtain reliable experimental data at high Mach numbers $(M>5$, hypersonic flow), but also experiments at moderate Mach numbers suffer from a strong dependence on the measurement instruments and therefore cannot describe with reasonable reliability the complex interaction of shock waves with turbulence.

Recently, the increases in computer power made it easier to set up numerical models, but problems remain, because, when dealing with turbulence, it is necessary to introduce closure conditions, which in turn are dependent on experimental data. For a review, see Andreopulos et al. (2000), Lele (1994), Adamson \& Messiter (1980), and references therein.

However, there is a general consensus that the most important feature of the interaction between shock waves and turbulence under unsteady conditions is the amplification of speed fluctuations and a strong change in the scale lengths, even though it is not well understood how this amplification occurs (Andreopulos et al. 2000). According to actual studies, the amplification depends on the shock strength, the state of the turbulence, and its level of compressibility. With respect to the amplification of the turbulent kinetic energy, Rotman (1991) reports an increase of a factor 2-2.15, while for Jacquin et al. (1993) the amplification depends on the density ratio:

$$
\frac{k^{2}(t)}{k^{2}(0)}=\frac{2+\rho^{2}(t) / \rho^{2}(0)}{3}
$$

where $k(t)$ and $\rho(t)$ are the turbulent kinetic energy and the gas density at the time $t$, respectively. In this case, the amplification of the kinetic energy can be up to $\sqrt{6}$ for monatomic gases (see the next section).

It is worth remembering that at high Mach number and for steady state motion, the effect of compressibility suppresses the turbulence and therefore there is no amplification. Changes in the Mach number (unsteady motion) generate distortions in shock waves, allowing the interaction with turbulence. The key point is therefore that the Mach number must not be constant. According to Jacquin et al. (1993), these conditions represent the "pressure released" regime and are the upper limit of the amplification. In this case, the turbulent Mach number can be higher than 1.

\section{Post-shock conditions}

For the purposes of the present note, we rearrange the Rankine-Hugoniot relations in the limit of a strong shock, that is with Mach number $M \gg 1$ (for reviews see, for example, Zel'dovich \& Raizer 1967; Landau \& Lifshitz 1987; 
Frank et al. 1992; Krolik 1999). The post-shock pressure $P_{2}$ is:

$P_{2} \approx\left(1-\frac{\rho_{1}}{\rho_{2}}\right)(1+\alpha) \rho_{1} V_{1}^{2}$

where $\alpha$ is the ionization factor ( $\alpha=1$ full ionization), $\rho_{2}$ is the post-shock density, $\rho_{1}$ and $V_{1}$ are the undisturbed density and speed of the plasma. Here, and in the following we use the subscripts 1 and 2 to indicate the undisturbed and the post-shock values, respectively.

The density ratio across the shock is given only once the value of the specific heat ratio $\gamma$ is known:

$\frac{\rho_{2}}{\rho_{1}} \approx \frac{\gamma+1}{\gamma-1}$.

From the equation of continuity across a shock, we can get easily that the ratio of velocities is a function of $\gamma$ only:

$\frac{V_{1}}{V_{2}}=\frac{\rho_{2}}{\rho_{1}} \approx \frac{\gamma+1}{\gamma-1}$.

In studies of AGN, velocities widths of typical bright BLR emission lines are non-relativistic, therefore $\gamma$ is generally considered to be $5 / 3$, the value of monatomic gas or metal vapors. Therefore, by considering $\gamma=5 / 3$ and Eq. (5), we obtain:

$P_{2} \approx 0.75(1+\alpha) \rho_{1} V_{1}^{2}$.

To calculate the post-shock temperature, we need of the equation of state:

$P=(1+\alpha) \rho R T$

where $R$ is the gas constant for the considered plasma. Therefore, by taking into account the Eqs. (5), (7), and (8), we can arrange the numerical value of the postshock temperature as a function of the undisturbed speed:

$T_{2} \approx \frac{2(\gamma-1)}{(1+\alpha) R(\gamma+1)^{2}} V_{1}^{2}$.

Please note that $V_{1}$ is given by Eq. (2), by considering the mean value. We could consider also the peak value, given by the sum of the average plus the fluctuating turbulent value.

\section{The broadening of emission lines}

There are several models to explain the broadening of emission lines by shocks (Perry \& Dyson 1985; CollinSouffrin et al. 1988; Fromerth \& Melia 2001). Recently, turbulent motion has been invoked to explain part of the broadening (Bottorff et al. 2000), even for Fe K $\alpha$ fluorescence line (Pairev \& Bromley 1998). Herein we apply turbulence amplification by shocks to investigate where in the range 0 to $10^{3} \mathrm{~km} \mathrm{~s}^{-1}$ (Bottorff et al. 2000) turbulence (if it exists in AGN) may lie.

As the electron temperature of the environment plasma was measured at $10^{4} \mathrm{~K}$, if we assume that protons have the same temperature of electrons, this implies a maximum fluctuation speed of some tens of $\mathrm{km} \mathrm{s}^{-1}$ (we have considered that under normal conditions the turbulence is limited by the local speed of sound; cf. Balbus \& Hawley 1998).

On the other hand, we can assume that the electron temperature is not representative of the mean temperature of the gas. If we consider a shock due - for example - to a wind with speed, say $500-1000 \mathrm{~km} \mathrm{~s}^{-1}$, the post-shock temperature, with reference to Eq. (9), is about 1.1-4.6× $10^{7} \mathrm{~K}$ (assuming $\alpha=0.5$ and a hydrogen plasma). This is the ion temperature, while the electron value is scaled by a factor $m_{\mathrm{e}} / m_{i} \approx 5.4 \times 10^{-4}\left(T_{\mathrm{e}} \approx 0.6-2.5 \times 10^{4} \mathrm{~K}, \mathrm{a}\right.$ value comparable to the observations). Indeed, it is known that the basic feature of shock waves in plasma is the slow energy exchange between ions and electrons, and the high electron mobility. The equilibrium temperature will be reached slowly after a time $t_{\mathrm{eq}}$ :

$\frac{\mathrm{d} T_{\mathrm{e}}}{\mathrm{d} t}=\frac{T-T_{\mathrm{e}}}{t_{\mathrm{eq}}}$

because the energy exchange occurs by Coulomb scattering and there is a large difference in mass between ions and electrons. These relaxation processes determine the thickness of the shock front (Zel'dovich \& Raizer 1967).

From the post-shock temperature, we can calculate the most probable thermal speed, assuming a Maxwellian distribution. Indeed, even though the electrons and ions do not exchange energy among them, they independently and quickly reach the Maxwellian distribution (generally after one collision). So, the most probable speed is:

$V_{\mathrm{th}}=\sqrt{\frac{2 k T}{m_{i}}}$

and the value of the turbulent speed, that is limited by the local sound speed (cf. Balbus \& Hawley 1998):

$u=c_{\mathrm{s}}=\sqrt{\frac{\gamma k T}{m_{i}}}$.

If the shock interacts with the turbulence under unsteady conditions, we have the amplification of the turbulent speed. We know from Sect. 3, the amplification of the kinetic energy, from which we can calculate the amplified speed, under the simplified assumption that the distortion affects only the speed and not the density. We introduce the speed amplification factor $\beta$ that is simply the square root of the factor for the kinetic energy. According to the theory by Rotman (1991), $\beta=\sqrt{2}$, while for Jaquin et al. (1993) it depends on the density ratio across the shock:

$\beta=\sqrt[4]{\frac{2+\rho_{2}^{2} / \rho_{1}^{2}}{3}}$

and can reach the value of 1.57 for a monatomic gas.

Therefore, the maximum value of amplified turbulent speed is:

$u_{\mathrm{a}}=\beta \sqrt{\frac{\gamma k T}{m_{i}}}$. 


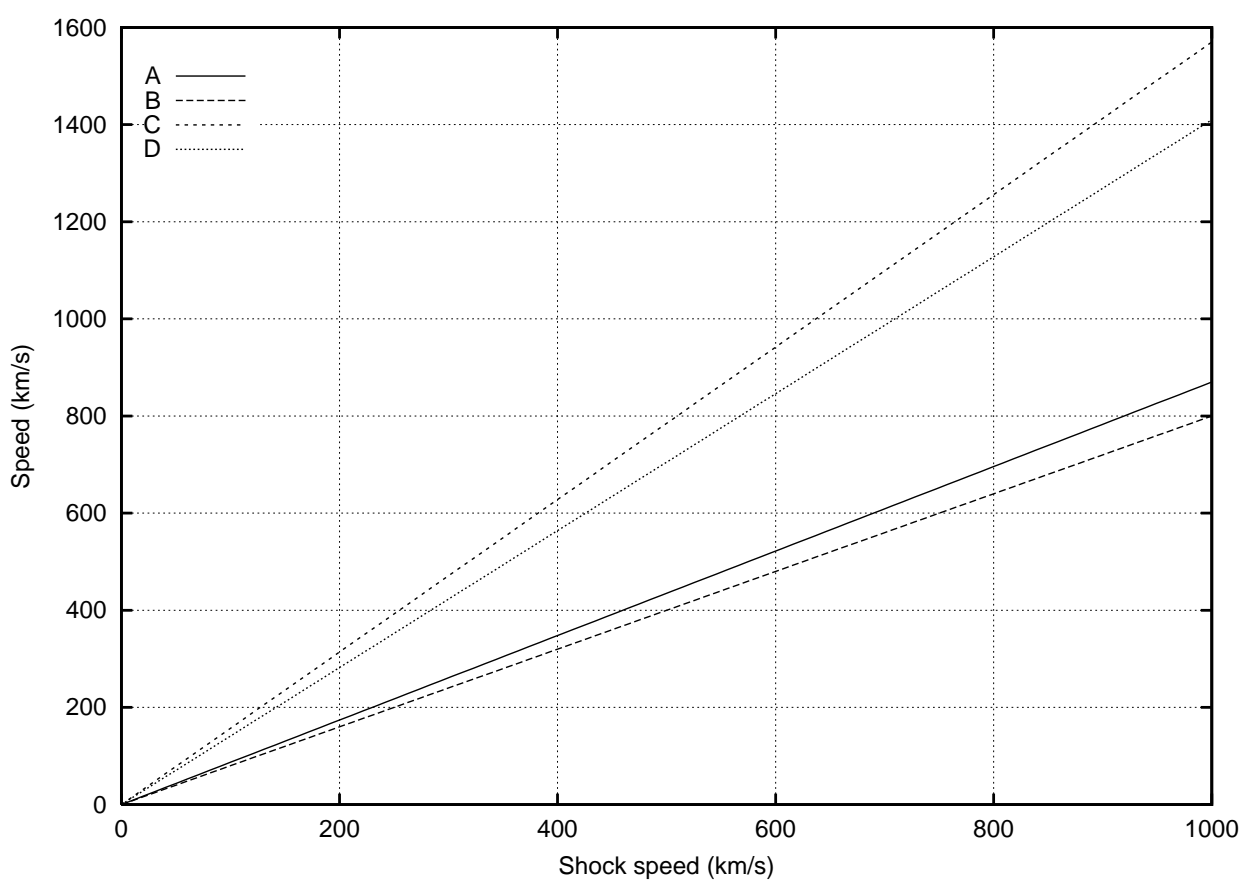

Fig. 1. Evaluation of post-shock thermal (A), turbulent (B), and amplified turbulent speed (C, according to Jaquin et al. (1993); D, according to Rotman (1991)) as a functions of the incoming shock speed.

Substituting Eq. (9) in Eqs. (11), (12), and (14), we obtain that $V_{\mathrm{th}} \approx 0.87 V_{1}, u \approx 0.8 V_{1}$, and $1.41 V_{1} \leq u_{\mathrm{a}} \leq 1.57 V_{1}$, according to the theory by Rotman or Jaquin et al., respectively. Some order-of-magnitude calculations are shown in Fig. 1.

The maximum value of the Doppler broadening $\Delta \nu / \nu_{0}$ of the emission lines due to the combined action of thermal and amplified turbulent speed is (see Rybicki \& Lightman 1979):

$\frac{\Delta \nu}{\nu_{0}}=\frac{1}{c} \sqrt{\frac{k T}{m_{i}}\left(2+\beta^{2} \gamma\right)}$

where $c$ is the speed of light. An evaluation of the influence of the amplification of turbulence on the Doppler broadening is shown in Fig. 2 for hydrogen plasma.

We can also do the reverse calculation, i.e. starting from post-shock temperature we can infer the speed. For example, we know that bright emission lines stop forming efficiently when $T \approx 10^{5} \mathrm{~K}$. For a fully ionized ( $\alpha=1)$ hydrogen plasma, Eq. (9) gives the pre-shock speed, which has the values of about $94 \mathrm{~km} \mathrm{~s}^{-1}$. For the amplified turbulent speed, we obtain a value between 133 and $148 \mathrm{~km} \mathrm{~s}^{-1}$, according to Rotman or Jaquin's theory, respectively. These values can be lower for lower ionization: if $\alpha=0.5$, we have that the mean value of the speed is between 115 and $128 \mathrm{~km} \mathrm{~s}^{-1}$. Note that values change if we deal with heavy ions.

Several authors have computed models for spectra from shocks (e.g. Cox 1972; Daltabuit et al. 1978; Shull \& McKee 1979; Binette et al. 1985) under the assumptions of steady state and constant pressure in the post-shock region. These conditions do not hold anymore in unsteady state and it would be necessary to set up a numerical model to evaluate the impact of the amplification of the turbulence on the emitted spectrum. However, we can now make some inferences: radiation generated into the shock acts to smooth the amplification, so as to establish again a sort of equilibrium. We know that hydrogen line intensities are very nearly proportional to $\rho v^{3}$ (Cox 1972). In this note, we assumed, for sake of simplicity, that turbulence affects only the speed and not the density, so we expect an increase of the hydrogen line intensities due to the amplification of the turbulent speed. Moreover, because the post-shock temperature can be high enough $\left(\approx 10^{7} \mathrm{~K}\right)$, we expect also an increase of $\mathrm{X}$-rays emission by thermal bremsstrahlung (if the shocked plasma is thin; otherwise a blackbody temperature is detected).

It is worth noting, that these are order-of-magnitude calculations made with the speed modulus, but we can observe and measure the radial component of these vectors. So we would introduce in calculations also the line-ofsight angle.

\section{Final remarks}

We emphasize that the purpose of this research note is to settle basic concepts about the turbulence amplification in AGN and some of the consequences, mainly related to the broadening of emission lines. Although in this case it is an additional term only, the impact of this physical mechanism on the observations is still to be determined, particularly with reference to LINER nuclei (cf. for example, Dopita et al. 1997; Allen et al. 1999; Evans et al. 1999).

The theory suffers from the lack of understanding of how amplification occurs - as underlined by several other 


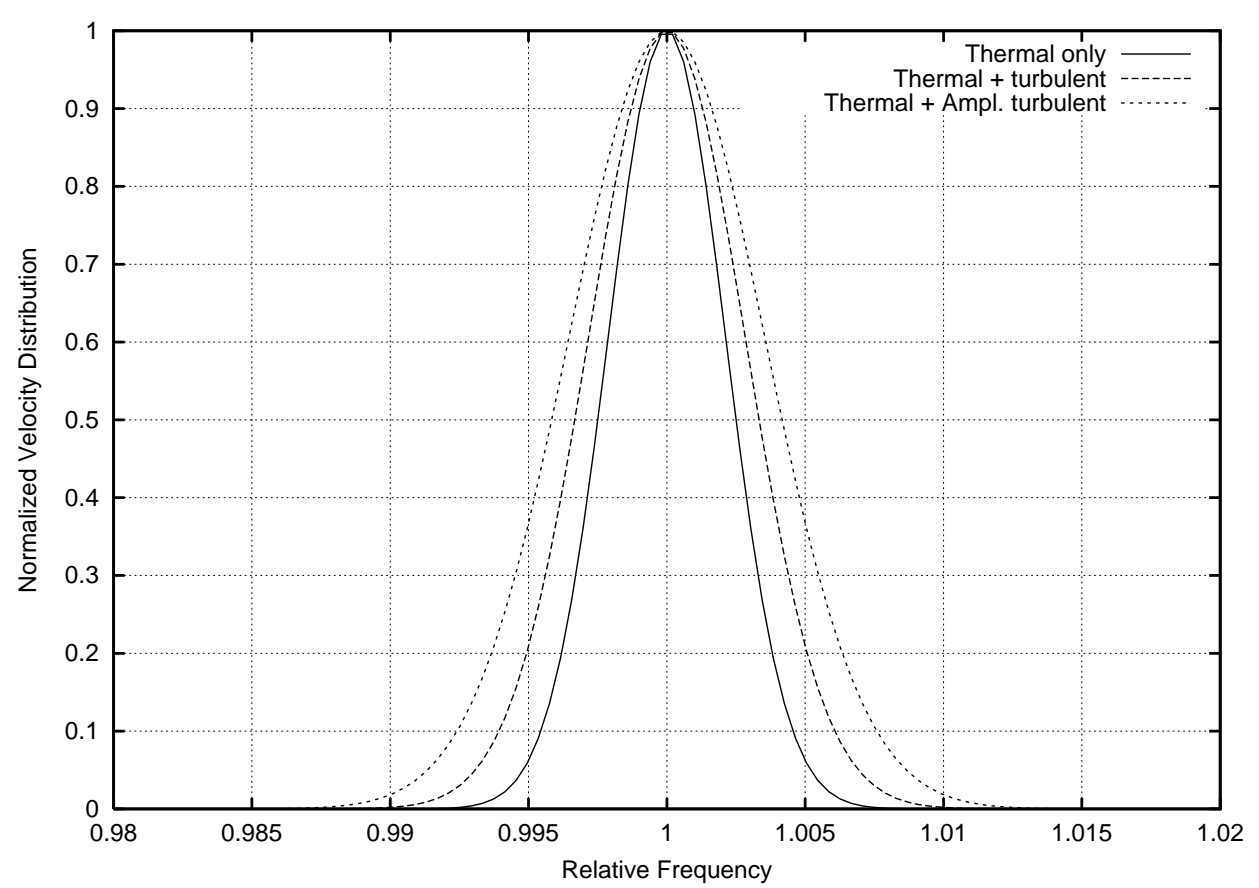

Fig. 2. Evaluation of broadening due to thermal speed only, additioned with turbulence, and amplified turbulence (according to Jaquin et al. 1993, i.e. the upper limit). Values are calculated for an incoming speed of $1000 \mathrm{~km} \mathrm{~s}^{-1}$.

authors - and therefore, for this application, observations of some AGN in soft X-rays or EUV are required to better assess the problem.

Acknowledgements. During early stage of this research, I have appreciated helpful suggestions in bibliographical search by J. Frank and useful discussions with N. Masetti. In addition, I wish to thank M. Cappi and M. Dadina for stimulating discussions, and J. B. Stephen for a critical review of the manuscript. I thank the four referees for their useful comments, which have helped me to improve this note. This research has made use of NASA's Astrophysics Data System Abstract Service.

\section{References}

Adamson, T. C., \& Messiter, A. F. 1980, Ann. Rev. Fluid Mech., 12, 103

Allen, M. G., Dopita, M. A., Tsvetanov, Z. I., et al. 1999, ApJ, 511,686

Andreopulos, Y., Agui, J. H., \& Briassulis, G. 2000, Ann. Rev. Fluid Mech., 32, 309

Balbus, S. A., \& Hawley, J. F. 1998, Rev. Mod. Phys., 70, 1

Baldwin, J. A., Ferland, G. J., Korista, K. T., et al. 1996, ApJ, 461,644

Binette, L., Dopita, M. A., \& Tuhoy, I. R. 1985, ApJ, 297, 476

Bottorff, M., Ferland, G., Baldwin, J., et al. 2000, ApJ, 542, 644

Bottorff, M., \& Ferland, G. 2002, ApJ, in press

Choudhuri, A. R. 1998, The physics of fluids and plasmas (Cambridge University Press, Cambridge)

Colbert, E. J. M., Baum, S. A., O'Dea, C. P., et al. 1998, ApJ, 496, 786

Collin-Souffrin, S., Dyson, J. E., McDowell, J. C., et al. 1988, MNRAS, 232, 539

Cox, D. P. 1972, ApJ, 178, 143
Daltabuit, E., Mac Alpine, G. M., \& Cox, D. P. 1978, ApJ, 219,372

Davidson, K., \& Netzer, H. 1979, Rev. Mod. Phys., 51, 715

Dopita, M. A., Koraktar, A. P., Allen, M. G., et al. 1997, ApJ, 490, 202

Evans, I., Koraktar, A. P., Allen, M. G., et al. 1999, ApJ, 521, 531

Fabian, A. C., Iwasawa, K., Reynolds, C. S., et al. 2000, PASP, 112,1145

Frank, J., King, A., \& Raine, D. 1992, Accretion power in astrophysics (2nd ed.; Cambridge, Cambridge University Press)

Fromerth, M. J., \& Melia, F. 2001, ApJ, 549, 205

Gillet, D., Debiéve, J. F., Fokin, A. B., et al. 1998, A\&A, 332, 235

Horne, K. 1995, A\&A, 297, 273

Jacquin, L., Cambron, C., \& Blin, E. 1993, Phys. Fluids A, 5, 2539

Krolik, J. 1999, Active Galactic Nuclei (Princeton University Press, Princeton)

Landau, L. D., \& Lifshitz, E. M. 1987, Fluid Mechanics - Course of theoretical physics, vol. 6, 2nd edition, Butterworth-Heinemann, Oxford

Lele, S. K. 1994, Ann. Rev. Fluid Mech., 26, 211

Pariev, V. I., \& Bromley, B. C. 1998, ApJ, 508, 590

Perry, J. J., \& Dyson, J. E. 1985, MNRAS, 213, 665

Rotman, D. 1991, Phys. Fluids A, 3, 1792

Rybicki, G. B., \& Lightman, A. P. 1979, Radiative processes in astrophysics (Wiley, New York)

Shull, J. M., \& McKee, C. F. 1979, ApJ, 227, 131

Sulentic, J. W., Marziani, P., \& Dultzin-Hacyan, D. 2000, ARA\&A, 38, 521

Tidman, D. A., \& Krall, N. A. 1971, Shock waves in collisionless plasmas (Wiley, New York)

Zel'dovich, Y. B., \& Raizer, Y. P. 1967, Physics of shock waves and high-temperature hydrodynamic phenomena, vol. II (Academic Press, New York) 